\title{
Hidden Markov models: an insight
}

\begin{abstract}
Hidden Markov models (HMM) is a probabilistic model consisting of variables representing observations, variables that are hidden, the initial state distribution, transition matrix, and parameters for all observation distributions. The said model is commonly used in speech recognition field and it has seen an increase in terms of usage, which include user profiling in mobile communication networks, and energy disaggregation. This paper shows, via numerical example, the computation of HMM's forward procedure will exceed the precision range of essentially any machine (even in double precision). It also extends the procedure to include Gaussian mixture hidden Markov models (GMHMM), the procedure that can be used as both a generator of observations, and as a model for how a given observation sequence was generated by an appropriate HMM.
\end{abstract}

Keyword: Baum-Welch/Expectation Maximization algorithm; Forward and backward procedures; Gaussian mixture hidden Markov models (GMHMM); Hidden Markov models (HMM); Simulation procedure 